\title{
Hand function of C6 and C7 tetraplegics 1-16 years following injury
}

\author{
LA Harvey*,1, J Batty $^{2}$, R Jones ${ }^{3}$ and J Crosbie ${ }^{1}$ \\ ${ }^{1}$ School of Physiotherapy, University of Sydney, Australia; ${ }^{2}$ Department of Physiotherapy, The Prince Henry \\ Hospital, Australia; ${ }^{3}$ Rehabilitation and Spinal Medicine, The Prince Henry Hospital, Australia
}

\begin{abstract}
Study design: Retrospective cohort study.
Objective: To quantify the hand function of C6 and C7 tetraplegics 1-16 years after injury. Setting: Patients were assessed in their homes.

Methods: Medical records of patients admitted to the Prince Henry Hospital Spinal Injuries Unit between 1984 and 1999 were used to identify all patients with C6 or C7 tetraplegia at 3 months post injury. Sixty-five patients (107 hands) were identified in this way. Forty-seven patients ( 81 hands) were located and agreed to partake in the study, and seven (nine hands) had died. Thus $81 \%$ of patients $(83 \%$ of hands) still alive at follow-up were assessed.

Main outcome measures: Unilateral hand function was assessed with the Grasp and Release Test (GRT) and a 10 item Activities of Daily Living (ADL) Test. Prevalance and severity of contractures, lateral grasp (key grip) strength and extensibility of the extrinsic finger flexor muscles were also determined.

Results: All hands except one had been managed without surgical intervention. The median number of ADL tasks successfully completed was $9 / 10$ (interquartile range $=8-10$ ) and the median number of objects successfully manipulated in the GRT was 3/6 (IQ range $=3-5$ ). Lateral grasp was poor $(74 \%$ and $75 \%$ of hands could not use a lateral grasp to move the paperweight or depress the fork in the GRT, respectively), and the prevalence of contractures was low (53\% of hands had full passive range of motion).

Conclusion: In the long term, most $\mathrm{C} 6$ and $\mathrm{C} 7$ tetraplegics attain a high level of hand function despite poor lateral grasps.

Spinal Cord (2001) 39, 37-43
\end{abstract}

Keywords: tetraplegia; upper limb function; hand; paralysis; rehabilitation

\section{Introduction}

C6 and C7 tetraplegics have paralysis of finger and thumb flexor muscles but retain voluntary control of wrist extensor and sometimes wrist flexor and finger extensor muscles. ${ }^{1,2}$ Despite such extensive paralysis these patients are generally able to hold and manipulate objects. ${ }^{3}$ An ability to effectively use the hand is critical to independence and quality of life. ${ }^{4,5}$ Consequently a great deal of attention is directed at optimising hand function of tetraplegics.

In the past, C6 and C7 tetraplegics' hands were managed with manual therapeutic regimes involving the administration of regular passive movements and stretches and the early application of splints. ${ }^{6-9}$ In addition or alternatively, patients were provided with orthotics and adaptive equipment. ${ }^{1,7,10}$ In more recent years surgery has been widely advocated as the treatment of choice, ${ }^{5,11-14}$ though a large proportion of patients are either unsuitable for surgery, do not

*Correspondence: LA Harvey, School of Physiotherapy, University of Sydney, P.O. Box 170, Lidcombe, NSW, 1825, Australia. have access to these types of service, or opt for nonsurgical interventions. Neuroprostheses are also occasionally used to enhance the hand function of this group of patients, ${ }^{4,15,16}$ though less commonly in C7 tetraplegics.

Surprisingly, there is little fundamental information about the hand function of $\mathrm{C} 6$ and $\mathrm{C} 7$ tetraplegics. Most studies either measure hand function following surgery $2,11,12,17-19$ or implantation of neuroprostheses. ${ }^{15,20,21}$ Alternatively, studies outline the principles underlying different methods of conservative , $^{1,7-9,22}$ and surgical management. ${ }^{2,5,23,24}$ Whilst some studies provide more quantitative information about the hand function of $\mathrm{C} 6$ and $\mathrm{C} 7$ tetraplegics, they recruit small numbers of subjects on the basis of convenience and consequently are susceptible to selection bias. ${ }^{25}$ No study has yet quantified the level of hand function attained by a large and representative cohort of $\mathrm{C} 6$ and $\mathrm{C} 7$ tetraplegics.

Quantitative information about the hand function typically attained by $\mathrm{C} 6$ and $\mathrm{C} 7$ tetraplegics is valuable for two reasons. Firstly, it enables therapists, clinicians 
and surgeons to better define 'good' and 'poor' outcomes. In this way, they are better equipped to objectively gauge the effectiveness of different interventions within the clinical setting. Secondly, information about the level of hand function typically attained is important for predicting hand function of tetraplegics. The ability to foresee future hand function enables therapists and clinicians to provide recently injured patients and their long-term care providers with accurate information about the level of hand function that can be expected.

The purpose of this study was to quantify the hand function of a large cohort of C6 and C7 tetraplegics long after injury. Specifically, the aims were to determine the effectiveness of palmar and lateral grasps, and assess abilities to use the hand to perform useful and purposeful unilateral tasks. Subjects in the cohort were managed conservatively (ie, without surgery) and thus the results of this study can be used to quantify the level of hand function that can be expected in the absence of surgical intervention.

\section{Methods}

\section{Subjects}

Medical records of patients admitted to the Prince Henry Hospital Spinal Injuries Unit in Sydney between 1984 and 1999 were examined. Patients were identified if during this period they had presented with a sudden onset of tetraplegia following trauma, surgery or a medical condition. All complete or incomplete C6 and C7 tetraplegics with grade $2 / 5$ or less strength in the finger flexor muscles at approximately 3 months after the onset of tetraplegia were included in the study (according to the American Spinal Injuries Association's standards for neurological classification ${ }^{26}$ ). Strength at 3 months post-injury was used as a selection criterion, rather than strength immediately post-injury, to eliminate patients whose neurological status improved markedly in the first 3 months. Patients who had died or been transferred elsewhere within the first 3 months after injury were also excluded.

Sixty-five patients were identified in this way. All were male except eight. Both hands of 42 patients and one hand of 23 patients met the inclusion criteria (107 hands in total). The median (interquartile range) age at injury was 28 years $(19-37$ years). Forty-seven of the initial cohort of 65 patients were located and agreed to participate in the study. In 34 both hands were suitable for inclusion and in the other 13 only one hand was suitable for inclusion ( 81 hands in total). Seven patients (nine hands) had died (median and interquartile age at injury was 62 years and 34-67 years, and median and interquartile age at death was 69 years and $42-74$ years). The remaining patients had either moved overseas (six patients, 10 hands), were unable to be located (one patient, one hand) or declined to be involved (four patients, six hands). In this way $81 \%$ of patients ( $83 \%$ of hands) alive at follow-up were reviewed. The project was approved by the appropriate Ethics Committees and all participants signed an informed consent sheet. Subjects were assessed by one of two therapists at their place of residence.

\section{Measurement procedure}

Strength of lateral and palmar grasp Initially 'strength' of the lateral and palmar grasps was measured. Strength of the lateral grasp was measured with a modified pinch meter. ${ }^{21,27}$ Subjects were tested three times and the median score was recorded. Strength of the palmar grasp was assessed by determining the ability to hold different sized and weighted cylinders in the palm of the hand with the thumb adducted adjacent to the index finger (ie, the thumb was not hooked around the cylinders). Cylinders were $2.5,5$ or $7.5 \mathrm{~cm}$ in diameter and weighted $0.1,0.5,1$ or $2 \mathrm{~kg}$, thus there were 12 different cylinders. The cylinders were placed in subjects' hands by the assessor and subjects were required to hold the cylinders upright for $10 \mathrm{~s}$. Subjects were scored out of 12 according to the number of cylinders successfully held.

Passive range of motion Passive range of motion (ROM) of the wrist, metacarpophalangeal (MCP) and interphalangeal (IP) joints were measured with a goniometer and rated according to the passive range of motion of the least mobile joint. The convention used was:

- Full ROM, indicated full passive range of motion in wrist, MCP and IP joints.

- Minimal loss of ROM, indicated loss of passive range of motion in wrist, MCP or any IP joint of $15^{\circ}$ or less.

- Moderate loss of ROM, indicated loss of passive range of motion in wrist, MCP or any IP joint of $15-45^{\circ}$.

- Severe loss of ROM, indicated loss of passive range of motion in wrist, MCP or any IP joint of $45^{\circ}$ or more.

Passive flexion of the MCP and IP joints was measured with the wrist extended to reduce stretch on the extrinsic finger and thumb extensor muscles. In the same way passive extension of the MCP and IP joints was measured with the wrist flexed to reduce stretch on the extrinsic finger and thumb flexor muscles. In this way, joint range of motion measures were not overly influenced by the extensibility of multi-articular muscles such as the extrinsic finger flexor and extensor muscles. ${ }^{22,28}$

Extensibility of the extrinsic finger flexor muscles Measures of passive wrist extension with the MCP and IP 
joints maintained in extension were used to reflect the extensibility of the extrinsic finger flexor muscles. Provided the extrinsic finger flexor muscles are sufficiently stretched over the MCP and IP joints of the fingers, they, rather than other muscles and soft tissue spanning the flexor aspect of the wrist, will predominantly limit wrist extension. ${ }^{29}$ In this way limited passive wrist extension reflects limited extensibility in the extrinsic finger flexor muscles and viceversa. Passive wrist extension was measured with a device previously described and tested for its reliability. ${ }^{29}$ A $0.72 \mathrm{Nm}$ wrist extension torque was applied and wrist angle was measured from a goniometer attached to the side of the measuring device. Zero degrees indicated a neutral position of the wrist and limited extensibility of the extrinsic finger flexor muscles.

Lateral and palmar grasp The effectiveness of lateral and palmar grasps was assessed with the Grasp and Release Test (GRT). ${ }^{15,21,27}$ The GRT was designed primarily to assess the effectiveness of lateral and palmar grasps in C5 and C6 tetraplegics fitted with neuroprostheses. It assesses the ability to pick up, move and release six objects of varying sizes, weights and textures using a palmar or lateral grasp. Each hand is tested and scored separately. The objects used in the GRT include a peg, paperweight, fork, block, can and videotape. Each object was chosen to represent one or more objects routinely manipulated for activities of daily living (ADL). For example, the paperweight, can and videotape were included to reflect subjects' abilities to hold large heavy objects such as a glass or book. The fork was included to not only assess subjects' ability to stab food but also their ability to grasp a pen. The fork was attached to a spring-loaded piston and subjects were required to hold the 'fork' and depress it downwards with a $4.4 \mathrm{~N}$ vertical force. Subjects were not permitted to depress the fork without grasping it between the thumb and index finger.

The GRT was administered according to conventional protoco $1^{15,21,30}$ and initially subjects were given a pre-test to determine which tasks they could and could not complete. Subjects were not tested on tasks that they were unable to perform in the pre-test. Subjects were given an opportunity to practise prior to testing and sufficient rest was provided between each item to minimise fatigue. The objects were presented in a random order and subjects were required to use either a palmar (for the block, video and can) or lateral (for the peg, fork and paper weight) grasp to move or depress each item. Subjects were scored on their ability to successfully move (or in the case of the fork, depress) each of the objects as many times as possible in $30 \mathrm{~s}$. In addition, a summed score (tallied GRT score) for each subject was calculated by tallying scores across items. If a subject failed to move an item in the pre-test they scored zero for that particular item.
Ability to use hand in any way to perform functional tasks An Activities of Daily Living Test (ADL test), similar to ones recommended by others, ${ }^{20,21,31-33}$ was also administered. It assessed subjects' ability to independently perform 10 unilateral tasks, namely using a fork, applying toothpaste to a toothbrush, answering a phone, writing with a pen, lifting a glass and mug to the mouth and picking-up a biscuit, toothbrush, computer disc and book. Unlike the GRT, subjects were allowed to use any method to perform each task (ie, they were not solely restricted to a lateral or palmar grasp). In this way, the test assessed the ability of subjects to use their hands in any way to perform purposeful tasks. However, subjects were not allowed to use splints, their other hand, another part of their body or adapted equipment.

The validity, reliability and sensitivity of the GRT and the ADL test have not been formally assessed. However, both were initially designed from existing hand assessment tools ${ }^{15,34}$ after extensive review of all other available assessment tools (see reference ${ }^{15}$ for summary of problems associated with using either the Sollerman or Jebsen tests for assessing tetraplegic hand function). The GRT and ADL test utilise objects commonly used in activities of daily living, so have face and content validity. In addition, there is some evidence to indicate that the GRT and similar activities of daily living tests are sensitive to change in function, especially following implantation of neuroprostheses.

\section{Data reduction and analysis}

Median and interquartile ranges were derived for all variables unless otherwise specified. In subjects with both hands included in the study, the results of each hand were analyzed separately. This was necessary because often subjects had asymmetrical lesions. In order to avoid confusion arising from this method of data analysis, most results are presented in terms of hands rather than subjects.

The neurological status of hands at follow-up were categorised into the following three groups:

(i) complete and incomplete C6 lesion with less than grade $3 / 5$ strength in the finger and/or thumb flexor muscles (C6 subjects).

(ii) complete and incomplete $\mathrm{C} 7$ lesion with less than grade $3 / 5$ strength in the finger and/or thumb flexor muscles (C7 subjects).

(iii) complete and incomplete $\mathrm{C} 6$ to $\mathrm{C} 8$ lesion with grade $3 / 5$ or more strength in the finger and/or thumb flexor muscles (finger/thumb flexor subjects).

In addition, for descriptive purposes, subjects' hands were also classified according to Moberg's modified International Classification for Surgery of the Hand in Tetraplegia (ICSHT ${ }^{2,34}$ ). 


\section{Results}

The median age at time of injury (and interquartile range) of the 47 reviewed subjects (81 hands) was 29 years (19-37 years; see Table 1). Subjects were reviewed on average (SD) 8 years since injury (5 years). Table 1 provides information about the neurological status of subjects' hands at follow-up according to the classifications of ASIA and ICSHT. At follow-up 62 hands $(77 \%)$ had either complete or incomplete C6 and C7 lesions with less than grade 3/5 strength in the finger and thumb flexor muscles. Whilst no hand of any subject had more than grade $2 / 5$ strength in the finger or thumb flexor muscles at 3 months post injury, 19 hands $(23 \%)$ had grade $3 / 5$ or more strength in these muscles at follow-up. Only one hand of a subject had deteriorated neurologically. This subject's hand had motor and sensory loss consistent with a complete C6 lesion at 3 months post injury (ie, wrist extension strength was $3 / 5$ ) but consistent with a complete C5 lesion at follow-up (ie, wrist extension strength was 3-/5). At the risk of inaccuracy but for the purpose of simplicity his lesion was classified as C6 for all analyzes.

All subjects' hands but one were conservatively managed with conventional occupational therapy and physiotherapy. ${ }^{22}$ The majority of subjects wore splints for between 2 and 4 months after injury but were not receiving any therapy (from therapists or carers) at follow-up. However, most stated that they themselves moved or stretched their hands regularly for therapeutic purposes. One subject with a bilateral C6 motor-complete lesion had undergone tendon transfers (brachioradialis to flexor pollicis longis and posterior deltoid to triceps) in one extremity, 1 year after injury. Whilst at follow-up his lateral grasp was relatively strong $(8.3 \mathrm{~N})$, his hand function was not dissimilar to his C6 counterparts (for example, he could successfully manipulate nine of the 10 objects in the ADL test and his tallied GRT score was 34). This subjects' data were included in all analyses.

Passive range of motion Only 16 hands (of nine subjects) had 'moderate' or 'severe' loss of passive range of motion (see Table 1) and more than half the hands (ie, 52\%) had full passive range of motion. Passive range of motion in the elbow and shoulder were not measured, however it was noted that three subjects (three hands) had severe contracture in one or both of these joints preventing reaching to the face and/or the testing table.

Strength of lateral and palmar grasp and extensibility of the extrinsic finger flexor muscles Extensibility of the extrinsic finger flexor muscles and 'strength' of the lateral and palmar grasps are presented in Table 1. Not surprisingly, subjects with paralysis of the finger and/or thumb flexor muscles had weaker lateral and palmar

Table 1 Characteristics of hands at follow-up including median (interquartile range) age at time of injury (years), time since injury (years), classification of hands according to ICSHT, lateral pinch strength (N), cylinder tally (number of cylinders held), passive wrist extension with MCP and IP extension, and prevalence of contractures. Contractures prevented testing of the extensibility of the extrinsic finger flexor muscles in 11 hands. For the purpose of analyses subjects' hands were classified into three groups, namely complete and incomplete C6 lesions with less than grade $3 / 5$ strength in finger and thumb flexor muscles, complete and incomplete $\mathrm{C} 7$ lesions with less than grade $3 / 5$ strength in finger and thumb flexor muscles and complete and incomplete $\mathrm{C} 6-8$ lesions with less than grade $3 / 5$ strength in finger and thumb flexor muscles

\begin{tabular}{|c|c|c|c|}
\hline & $\begin{array}{c}\text { C6 with }<\text { grade } 3 / 5 \\
\text { finger } / \text { thumb flexion } \\
(n=38)\end{array}$ & $\begin{array}{c}\text { C7 with }<\text { grade } 3 / 5 \\
\text { finger } / \text { thumb flexion } \\
(n=24)\end{array}$ & $\begin{array}{c}\text { C6 }- \text { C8 with }<\text { grade } 3 / 5 \\
\text { finger } / \text { thumb flexion } \\
(n=19)\end{array}$ \\
\hline Age at time of injury & $31(19-33)$ & $24(19-31)$ & $56(29-57)$ \\
\hline Time since injury & $10(4-14)$ & $8(4-10)$ & $6(4-12)$ \\
\hline $\begin{array}{l}\text { Classification of hands } \\
\text { according to ICSHT }\end{array}$ & $\begin{array}{c}\text { Cu. } 1=1^{\mathrm{a}}, \mathrm{O} .2 / 3=5 ; \\
\text { Cu. } 2 / 3=12^{\mathrm{b}}, \mathrm{O} .4=1 ; \\
\text { Cu. } 4=13^{\mathrm{c}}, \mathrm{O} .5=2 ; \mathrm{Cu} .5=4\end{array}$ & $\begin{array}{l}\text { O. } 2 / 3=1 ; \text { Cu. } 4=5 \\
\text { Cu. } 5=14 ; \text { Cu. } 6=4\end{array}$ & $\begin{array}{c}\text { Cu. } 2 / 3=3 ; \text { Cu. } 4=4 ; \\
\text { Cu. } 5=5 ; \text { Cu. } 6=5^{\text {d }}, \\
\text { Cu. } 7=2\end{array}$ \\
\hline Lateral pinch strength & $3(1.9-5.8)$ & $4(2.5-6.7)$ & $17(12.5-30.8)$ \\
\hline Cylinder tally $(\max .=12)$ & $3(2-5)$ & $5(3-6)$ & $8(6-11)$ \\
\hline \multicolumn{4}{|l|}{ ROM: } \\
\hline Full passive range & 17 & 18 & 7 \\
\hline$<15^{\circ}$ loss & 13 & 5 & 3 \\
\hline $15-46^{\circ}$ loss & 5 & 1 & 3 \\
\hline$>45^{\circ}$ loss & 3 & 0 & 4 \\
\hline $\begin{array}{l}\text { Wrist extension with MCP } \\
\text { and IP extension }\end{array}$ & $85(80-90)$ & $80(75-85)$ & $75(60-75)$ \\
\hline Tallied GRT score & $35(20-53)$ & $47(34-57)$ & $70(53-100)$ \\
\hline
\end{tabular}

${ }^{a}$ One hand had grade 3-/5 strength in the wrist extensor muscles but was classified as ASIA C6 for simplicity (see text for details). ${ }^{b}$ One hand had been treated surgically (see text for details). ${ }^{\mathrm{c}}$ Two hands had severe elbow and/or shoulder contractures restricting upper limb movement between the testing table and hand (see text for details). ${ }^{\mathrm{d}}$ One hand had severe elbow and/or shoulder contractures restricting upper limb movement between the testing table and hand (see text for details) 
grasps than the $\mathrm{C} 6-\mathrm{C} 8$ subjects with grade $3 / 5$ or more strength in these muscles.

Lateral and palmar grasp The effectiveness of lateral and palmar grasps was measured with the GRT. Subjects that passed the pre-GRT rarely dropped or failed to successfully grasp an item during testing (median and interquartile failure rate was 0 and $0-1$ failures per tested item). Nearly all subjects could successfully move the peg and block, regardless of paralysis of the finger and thumb flexor muscles (Figures 1 and 2). The hands of subjects with grade $3 / 5$ or more strength in the finger and/or thumb flexor muscles were more successful at manipulating the paper weight, fork and video than the hands of subjects with paralysis in these muscles. In contrast, there was little difference in the ability of subjects to move the can.

The tallied GRT scores are provided in Table 1. Subjects with active finger or thumb flexion at followup attained higher tallied GRT scores than those without.

Ability to use the hand in any way to perform functional tasks The ADL test reflects subjects' ability to use their hands in any way to perform purposeful tasks. Figure 3 shows the percentage of hands that could successfully manipulate each of the 10 items. Subjects had particular difficulty with the fork, glass and pen. Figure 4 shows the correlation between tallied GRT scores and ADL test scores. There was a clear 'ceiling' effect with the ADL test, and a close but non-linear

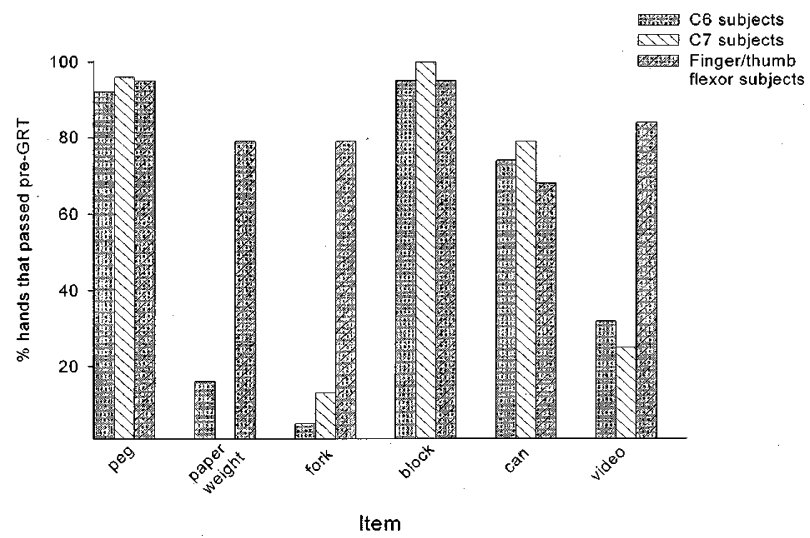

Figure 1 Percentage of hands that passed the initial preGRT (ie, successfully moved items in the GRT at least once with the required grasp). For the purposes of analyses subjects' hands were classified into three groups, namely complete and incomplete C6 lesions with less than grade 3/5 strength in finger and thumb flexor muscles (C6 subjects), complete and incomplete $\mathrm{C} 7$ lesions with less than grade $3 / 5$ strength in finger and thumb flexor muscles (C7 subjects), and complete and incomplete C6-C8 lesions with grade 3/5 or more strength in the finger and/or thumb flexor muscles (finger/thumb flexor subjects) correlation between the GRT and ADL test scores $($ Spearman's rho $=0.80)$.

\section{Discussion}

This study quantifies the hand function of $\mathrm{C} 6-\mathrm{C} 7$ tetraplegics $1-16$ years after injury. All subjects had at

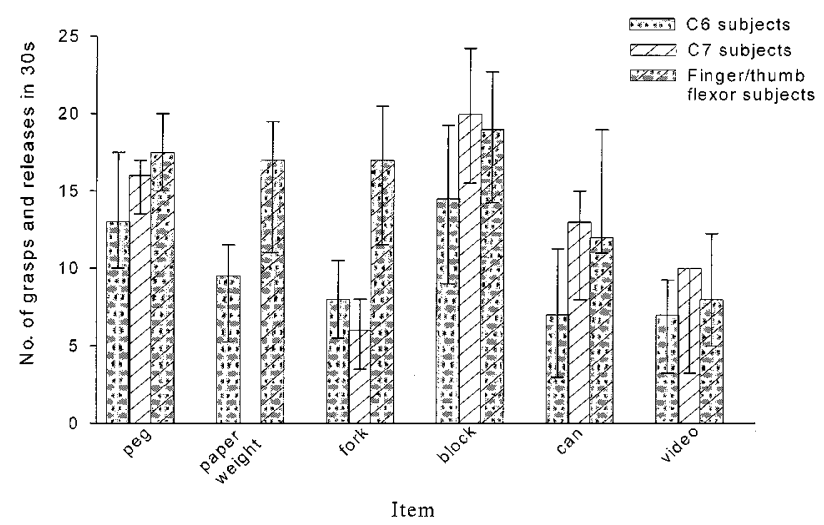

Figure 2 Median (and interquartile) number of successful grasps and releases in $30 \mathrm{~s}$ of the items in the GRT. Only the data of subjects that successfully passed the pre-test are included. For the purposes of analyses subjects' hands were classified into three groups, namely complete and incomplete C6 lesions with less than grade $3 / 5$ strength in finger and thumb flexor muscles (C6 subjects), complete and incomplete C7 lesions with less than grade $3 / 5$ strength in finger and thumb flexor muscles (C7 subjects), and complete and incomplete $\mathrm{C} 6-\mathrm{C} 8$ lesions with grade $3 / 5$ or more strength in the finger and/or thumb flexor muscles (finger/thumb flexor subjects)

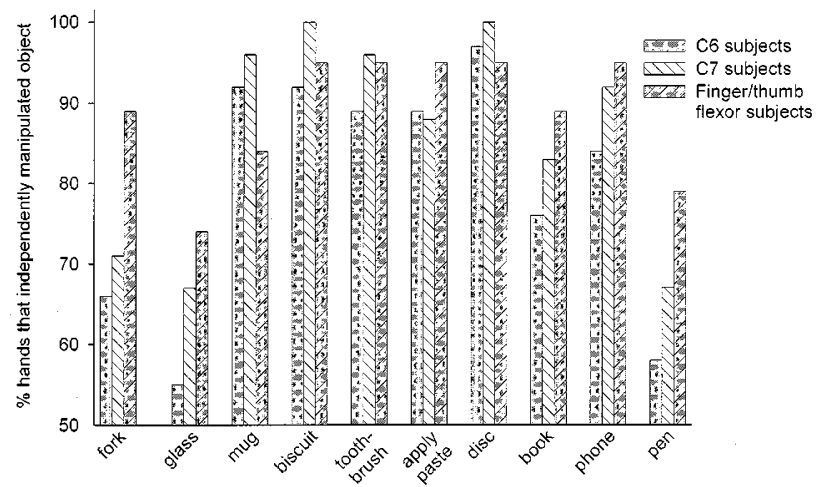

Figure 3 Percentage of hands that independently manipulated the different items of the ADL test. For the purposes of analyses subjects' hands were classified into three groups, namely complete and incomplete C6 lesions with less than grade $3 / 5$ strength in finger and thumb flexor muscles, complete and incomplete C7 lesions with less than grade $3 / 5$ strength in finger and thumb flexor muscles, and complete and incomplete $\mathrm{C} 6-\mathrm{C} 8$ lesions with grade $3 / 5$ or more strength in the finger and/or thumb flexor muscles 


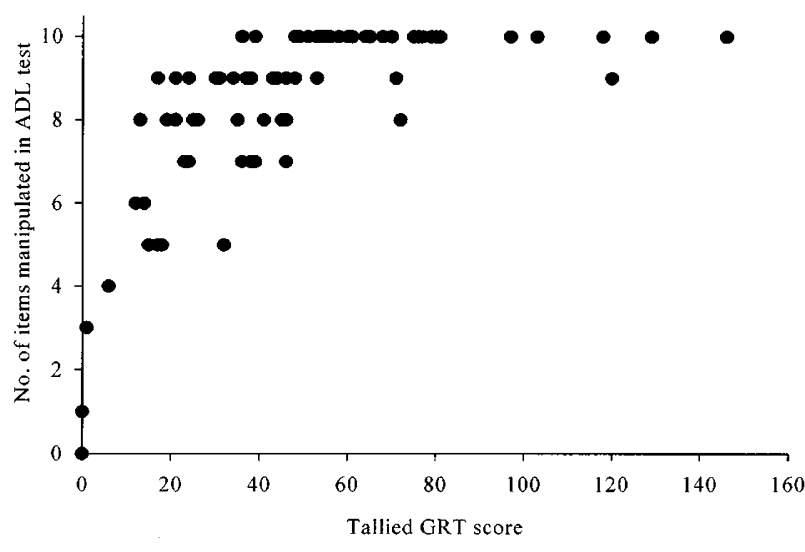

Figure 4 Correlation between tallied GRT score and number of items successfully manipulated in the ADL test

least grade $3 / 5$ wrist extension and not more than grade $2 / 5$ finger or thumb flexion at 3 months post injury and only one subject had undergone surgical intervention. Previous studies have also provided descriptive information about the hand function of C6 and C7 tetraplegics managed conservatively, ${ }^{7,25}$ but such studies have recruited small numbers of subjects on the basis of convenience and have consequently been susceptible to selection bias. Bias of this type was minimised in this study by assessing $81 \%$ of a consecutive series of eligible C6 and C7 tetraplegics still alive at follow-up.

The ADL test assessed the ability of subjects to perform important activities of daily living. The results indicated that subjects attained high levels of hand function without surgical intervention and despite extensive paralysis. For example, $71 \%$ of the C6 hands, $79 \%$ of the $\mathrm{C} 7$ hands and $89 \%$ of $\mathrm{C} 6-\mathrm{C} 8$ hands with active finger and/or thumb flexion could independently perform at least eight of the 10 tasks. Even higher levels of hand function would have been attained if subjects were not restricted to using one hand.

Often subjects used novel and ingenious ways to perform the tasks of the ADL test without utilising lateral or palmar grasps. $^{25}$ For example, subjects unable to use a lateral grasp to pick up the book often were able to pick it up between the second and third finger or rotate it over and cradle it in the palm of the hand. Similarly subjects unable to use a lateral grasp to hold the fork or pen could weave these utensils over and under fingers.

Currently there is no standardised or accepted tool for assessing tetraplegics' ability to use their hands in a functional way, ${ }^{1,6,9,15,35}$ though such a tool is urgently needed. The ADL test used in this study was similar to ones previously used to assess the effectiveness of neuroprostheses, ${ }^{20,21,31-33}$ and while it provided valuable information about subjects' abilities to perform important activities of daily living, there were some difficulties in differentiating subtle differences in function of the more capable subjects. These problems could be somewhat overcome either by changing the scoring system to account for differences in the time taken to complete each task or by including some more challenging manipulative tasks.

The effectiveness of the lateral and palmar grasp was measured with the GRT (the peg, paper weight and fork items were used to assess lateral grasp and the block, can and video items were used to assess palmar grasp). Subjects with paralysis of the finger and/or thumb flexor muscles were generally unable to hold the fork or paperweight between the thumb and index finger. Similarly they were unable to use a palmar grasp to pick up and move the video, though they had little difficulty with the block and can items. The few subjects with paralysis of the finger and/or thumb flexor muscles who successfully picked up these items often enhanced their grasps by eliciting spasticity in the finger and thumb flexor muscles. Interestingly, subjects with active finger and/ or thumb flexion also had difficulty completing the can task. This was perhaps because four subjects (seven hands) had unusual patterns of neurological loss with active finger flexion but paralysis of the finger extensor muscles. These patients tended to have contractures of the IP joints and/or excessive loss of extensibility in the finger flexor muscles, making it difficult for them to initially get their fingers around the can.

The incidence of contracture was low. Some subjects with moderate or severe contractures at follow-up attained high tallied GRT and ADL test scores. One notable subject with active finger and thumb flexion had severe contractures in his hand yet independently manipulated nine of the 10 items in the ADL test and attained a tallied GRT score of 79 .

Currently there is little consensus about optimal management of the $\mathrm{C} 6$ and $\mathrm{C} 7$ tetraplegic hand. Whilst surgery is widely advocated, its benefits have not been clarified with good quality randomised clinical trials. Similarly, no splinting or manual therapy protocol has been subjected to rigorous evaluation. This is in part due to the inherent difficulties associated with performing good quality research in this area. The results of the present study provide baseline information on the hand function of C6 and C7 tetraplegics managed without surgery $1-16$ years after injury. This information can be used by researchers to quantify the effectiveness of different interventions. In addition, this information can be used by clinicians to monitor outcomes and objectively gauge the effectiveness of different interventions on a day-to-day basis.

\section{Acknowledgements}

We acknowledge the financial assistance provided by the Motor Accident Authority of NSW. 


\section{References}

1 Sutton S. An overview of the management of the C6 quadriplegic patient's hand: an occupational therapist's perspective. $\mathrm{Br} J \mathrm{Occ}$ Ther 1993; 56: $376-380$

2 House JH. Reconstruction of the thumb in tetraplegia following spinal cord injury. Clin Orthop Rel Res 1985; 195: 117-128.

3 Peckham PH, Marsolais E, Mortimer JT. Restoration of key grip and release in the C6 tetraplegic patient through functional electrical stimulation. $J$ Hand Surg (Am) 1980; 5A: 462-469.

4 Mulcahey MJ et al. Functional neuromuscular stimulation: outcomes in young people with tetraplegia. J Am Para Soc 1994; 17: $20-35$.

5 Waters RL, Sie I, Gellman H, Tognella M. Functional hand surgery following tetraplegia. Arch Phys Med Rehab 1996; 77: $86-94$.

6 Curtin M. The management of the C6 quadriplegic patient's hand. (letter) Br J Occ Ther 1993; 56: 455.

7 DiPasquale-Lehnerz P. Orthotic intervention for development of hand function with C-6 quadriplegia. Am J Occ Ther 1994; 48: $138-144$.

8 Krajnik SR, Bridle MJ. Hand splinting in quadriplegia: current practice. Am J Occ Ther 1992; 46: 149-156.

9 Curtin M. Development of a tetraplegic hand assessment and splinting protocol. Paraplegia 1994; 32: 159- 169.

10 Nichols PJ, Peach SL, Haworth RJ, Ennis J. The value of flexor hinge and splints. Pros Orth Int 1978; 2: 86-94.

11 Gansel J, Wates R, Gellman H. Transfer of the pronator teres tendon to the tendons of the flexor digitorum profundus in tetraplegia. J Bone Joint Surg (Am) 1990; 72A: 427-432.

12 House JH, Comadoll J, Dahl A. One-stage key pinch and release with thumb carpal-metacarpal fusion in tetraplegia. J Hand Surg (Am) 1992; 17A: 530-538.

13 Freehafer AA. Tendon transfers in patients with cervical spinal cord injury. J Hand Surg (Am) 1991; 16A: 804-809.

14 Moberg E. Surgical rehabilitation of the upper limb in tetraplegia. Paraplegia 1990; 28: 330-334.

15 Wuolle KS et al. Development of a quantitative hand grasp and release test for patients with tetraplegia using a hand neuroprosthesis. J Hand Surg (Am) 1994; 19A: 209-218.

16 Keith $\mathrm{M}$ et al. Tendon transfers and functional electrical stimulation for restoration of hand function in spinal cord. $J$ Hand Surg (Am) 1996; 21A: 89-99.

17 Kelly CM, Freehafer AA, Peckman PH, Stroh K. Postoperative results of opponensplasty and flexor tendon transfer in patients with spinal cord injury. J Hand Surg (Am) 1985; 10A: 890-894.

18 Hentz VR, Brown M, Keoshian LA. Upper limb reconstruction in quadriplegia: functional assessment and proposed treatment modifications. J Hand Surg (Am) 1983; 8A: 119-131.
19 Vanden Berghe A, Van Laere M, Hellings S, Vercauteren M Reconstruction of the upper extremity in tetraplegia: functional assessment, surgical procedures and rehabilitation. Paraplegia 1991; 29: $103-112$.

20 Wijman CA et al. Functional evaluation of quadriplegic patients using a hand neuroprosthesis. Arch Phys Med Rehab 1990; 71: $1053-1057$.

21 Mulcahey MJ et al. Implanted functional electrical stimulation hand system in adolescents with spinal injuries: an evaluation. Arch Phys Med Rehab 1997; 78: 597-607.

22 Harvey L. Principles of conservative non-orthotic management for a tenodesis grip. $J$ Hand Ther 1996; 9: $238-242$.

23 Waters R, Moore KR, Graboff SR, Paris K. Brachioradialis to flexor pollicis longus tendon transfer for active lateral pinch in the tetraplegic. Hand Surg (Am) 1985; 10A: 385-391.

24 Moberg E. Surgical treatment for absent single-hand grip and elbow extension in quadriplegia. Principles and preliminary experience. J Bone Joint Surg (Am) 1975; 57A: 196-206.

25 Curtin M. An analysis of tetraplegic hand grips. Br J Occ Ther 1999; 62: $444-450$.

26 Ditunno JF, Young W, Donovan WH, Creasey G. The international standards booklet for neurological and functional classification of spinal cord injury. American Spinal Injuries Association. Paraplegia 1994; 32: $70-80$.

27 Smith BT, Mulcahey MJ, Betz RR. Quantitative comparison of grasp and release abilities with and without functional neuromuscular stimulation in adolescents with tetraplegia. Paraplegia 1996; 34: 16-23.

28 Keenan MA et al. Results of transfer of the flexor digitorum superficialis tendons to the flexor digitorum profundus tendons in adults with acquired spasticity of the hand. J Bone Joint Surg (Am) 1987; 69A: $1127-1132$.

29 Harvey L, King M, Herbert R. Reliability of a tool for measuring of long finger flexor muscles. $J$ Hand Ther 1995; 7: 251 - 254 .

30 Neurocontrol Corporation. Grasp-Release Test Kit Manual. Cleveland, Ohio, USA and Brussels, Belgium.

31 Saxena S, Nikolic S, Popovic D. An EMG-controlled grasping system for tetraplegics. J Rehab Res Dev 1995; 32: 17-24.

32 Stroh KC, Van Doren C. An ADL test to assess hand function with tetraplegic patients. J Hand Ther 1994; 7: 47-48.

33 Kilgore KL et al. An implanted upper-extremity neuroprosthesis: follow-up of five patients. J Bone Joint Surg (Am) 1997; 79A: $533-541$.

34 McDowell CL, Moberg EA, Smith AG. International conference on surgical rehabilitation of the upper limb in tetraplegia. $J$ Bone Joint Surg (Am) 1979; 4A: 387-390.

35 Lamb D. The current state of management of the upper limb in tetraplegia. Paraplegia 1992; 30: 65-67. 